\title{
Omission training compared with yoked controls and extinction in multiple-schedule discrimination learning*
}

\author{
CHARLES N. UHL and ANDREW L. HOMER \\ University of Utah, Salt Lake City, Utah 84112
}

\begin{abstract}
After rats were trained on a multiple schedule with variable interval 30 -sec reinforcement in both stimulus components, omission, yoked, and extinction procedures were applied in S- while the variable interval continued in $\mathbf{S}+$. In $\mathbf{S}-$, omission training reduced response rate faster and to a lower terminal level than either response-independent yoked reinforcement or extinction, which were approximately equivalent. In $\mathrm{S}+$, the extinction group exhibited elevated response rates representing behavioral contrast, while the omission and yoked groups showed reduced response rates. These results attest to the effectiveness of omission training as a response elimination method. They also contradict theories which posit reduction of response rate in $\mathrm{S}-$ to be necessary and sufficient for behavioral contrast.
\end{abstract}

Omission training, described by Grant (1964), is an operant procedure for eliminating behavior with positive reinforcement. Omission training is characterized by a response dependency in which reinforcement is omitted after a selected response, but reinforcement occurs if no response is performed for a prescribed length of time. Uhl and Garcia (1969) described two temporal parameters which identify the dependencies between behavior and reinforcement on an omission schedule: (a) a postresponse interval which sets the time that reinforcement is postponed (omitted) following a response, and (b) an interreinforcement interval which sets the time between consecutive reinforcements when no response intervenes.

There have been a number of demonstrations of the effectiveness of omission (OM) as a response elimination method (Davis \& Bitterman, 1971; Harman, 1973; Nevin, 1968; Smith \& Clark, 1972; Topping, Pickering, \& Jackson, 1971; Uhl, 1973; Uhl \& Garcia, 1969; Weisman, 1970; Zeiler, 1971). Comparisons of OM with extinction (EXT) have shown the two procedures to be approximately equal in response elimination effectiveness. Some studies have found response elimination to be more rapid with OM (Nevin, 1968; Snow \& Uhl, 1968; Topping \& Larmi, 1973; Zeiler, 1971), and some have found EXT somewhat more rapid (Harman, 1973; Uhl, 1973; Uhl \& Garcia, 1969; Uhl \& Sherman, 1971). Despite the equivocal data concerning the relative speed of response elimination with $O M$ vs EXT, the data are unanimous in demonstrating greater durability of OM effects (Harman, 1973; Topping \&

\footnotetext{
*This research was supported by Grant MH 13904 from the National Institute of Mental Health. The authors wish to thank Candace Lindsey for her assistance. Reprints may be obtained from C. N. Uhl, Department of Psychology. University of Utah, Salt Lake City, Utah 84112
}

Larmi, 1973; Uh1, 1973; Uhl \& Garcia, 1969; Uhl \& Sherman, 1971; Zeiler, 1971). Most tests of durability entailed a transition from OM or EXT to a response-independent variable time (VT) or fixed time (FT) reinforcement schedule. Introduction of a VT or FT schedule led to considerable response resumption after EXT but little resumption of responding after OM. The available data taken together, especially the durability data, attest to the effectiveness of $\mathrm{OM}$ as a method for achieving response elimination.

Most OM vs EXT comparisons have used nondiscriminative procedures. There are fragmentary data demonstrating $\mathrm{OM}$ effectiveness in discrimination learning in multiple schedules. Reynolds (1961) and Weisman (1970) obtained discrimination learning with $\mathrm{OM}$ in one component of a multiple schedule, but neither of these studies compared OM with other response elimination methods. Nevin (1968) and Snow and Uhl (1968) found more rapid discrimination learning with OM than with EXT in the S- (response elimination) component of a multiple schedule. However, response elimination data were not presented in detail in either of the latter studies, nor were any durability data reported.

The present experiment was designed to accomplish a detailed comparison between OM and EXT in multiple-schedule discrimination learning. The present design included a yoked control group in an attempt to clarify OM effects. Every OM S was run with a yoked (YOKE) partner which received reinforcement independently of its own behavior whenever its OM partner earned reinforcement. Such a control helps to separate the effects of the sheer receipt of reinforcement (by the YOKE S) from the effects of the dependency of reinforcement on not responding in an OM schedule.

All Ss were trained on a baseline multiple VI VI 
reinforcement schedule before a transition to response elimination in the S- component. In an attempt to provide an appropriate comparison with EXT, the temporal parameter values selected for the OM schedule were ones shown to be maximally effective in nondiscriminative procedures (Smith \& Clark, 1972; Uhl \& Garcia, 1969). Both of the OM intervals were made variable to minimize the possibility of discriminating between the S+ (VI) and S- (OM or EXT) components on the basis of the periodicity of reinforcers (cf. Jenkins, 1965). Because of some evidence (Kamil \& Davenport, 1968) that discrimination learning is facilitated by the interpolation of a time-out (TO) between stimulus components, half of the Ss in each of the present groups had a brief TO between components and half had no TO to further assess TO effects.

\section{METHOD}

\section{Subjects}

The Ss were 24 male albino rats $(250-399 \mathrm{~g})$ which were individually caged and maintained at $80 \%$ of their free-fceding weight throughout the experiment.

\section{Apparatus}

Experimentation took place in four identical operant boxes each equipped with a liquid dipper (.04-ml cup), a retractable lever, an overhead houselight, an exhaust fan, and a speaker providing $85-\mathrm{dB}$ masking white noise. Ambient illumination measured at the floor of the box when the houselight was on and off, respectively, was approximately 7.5 and $.5 \mathrm{fc}$.

All experimental events were controlled by and behavior was recorded by a NOVA digital computer (Data General Corporation) with a Sykes magnetic cassette tape recorder and a relay interface to the operant boxes. Each box was serviced every $10 \mathrm{msec}$ by the computer to time experimental events and to record responses. Data recording was made on magnetic tape for every response, reinforcement, or stimulus change event, together with the interevent time, to the nearest $10 \mathrm{msec}$.

\section{Procedure}

The rats were allowed free access to food for 2 weeks after arrival at the laboratory to establish a base weight, after which they were handled in pairs briefly for 5 days while they were reduced to $80 \%$ of base weight. Three daily $30-\mathrm{min}$ sessions of magazine training on a constant probability VT $30-\mathrm{sec}$ $(p=.0033$ per $t=.1 \mathrm{sec})$ reinforcement schedule followed. The reinforcer was $.04 \mathrm{ml}$ of a $30 \%$ (by weight) sucrose solution. The reinforcer event consisted of releasing the normally lowered arm of the dipper which made an audible "clack" sound when the cup became accessible to the rat. A pilot light inside the recessed circular housing where the dipper cup emerged was lit for the 3-sec duration of the reinforcer to provide an additional discriminative stimulus for the event. This describes the reinforcing event for the duration of the experiment. On the day following magazine training, leverpressing was manually shaped, then leverpresses were reinforced on a fixed ratio (FR) 1 for 40 reinforcements before changing to FR 2 for 40 more reinforcements.

Baseline Training. Baseline training was conducted for 10 daily 40-min sessions on a multiple VI $30-\mathrm{sec}$ VI $30-\mathrm{sec}$ reinforcement schedule. Both VIs were constant probability schedules $(\mathrm{p}=.0033$ per $\mathrm{t}=.1 \mathrm{sec})$. The two stimulus components of the multiple schedule consisted of houselight on and houselight off. Stimuli alternated for 1-min periods (20 periods of each per session) for the duration of the experiment.
Duration of stimulus periods did not include reinforcement time. A reinforcement made available but not collected at the end of a stimulus period remained available and could be collected when that same stimulus was presented the next time. For one group of $\mathrm{Ss}$ the transitions between stimulus periods was immediate, and for a second group there was an interpolated 10 -sec TO when the lever was retracted and the box was entirely dark. There was no opportunity for unreinforced responses before or after any sessions throughout the experiment since a session began with the insertion of the lever and onset of the white noise, and a session ended with retraction of the lever and offset of the white noise.

Discrimination Training. The Ss were quasi-randomly assigned to groups to begin the first discrimination training phase. There was a restriction that each operant box be equally represented in each experimental group. The experimental design for the discrimination phase was a 3 by 2 factorial incorporating, respectively: (a) EXT, OM, and YOKE reinforcement conditions in the houselight on component $(\mathrm{S}-$ ) to eliminate responding, and (b) TO or no TO between stimulus periods. The VI 30-sec continued with the houselight of $(\mathrm{S}+)$ for all groups. For the EXT group, no feature of the reinforcer event ever occurred during $S_{-}$. The OM schedule provided the reinforcer according to 10 -sec interreinforcement and $30-\mathrm{sec}$ postresponse variable intervals. Variable intervals were programmed by computing two sets of 30 times equally spaced between $.1 \mathrm{sec}$ and twice the mean interval value $(10$ or $30 \mathrm{sec})$. One of these times was selected at random from the appropriate set following a response (postresponse interval) or reinforcement (interreinforcement interval). The reinforcer was provided whenever the $O M$ interval in effect timed out. Note that reinforcers would occur once every $10 \mathrm{sec}$, on the average, if the OM $S$ did not respond in $S-$. A YOKE $S$ received the reinforcer at the same time as its OM partner regardless of its own behavior. Discrimination training continued for 30 daily sessions.

Durability Testing. Following discrimination training, two procedures to test the durability of response elimination effects in $\mathrm{S}-$ were begun. Half of the $\mathrm{Ss}$ in each group were assigned to EXT and half were assigned to a constant probability VT $30-\mathrm{sec}$ $(p=.0033$ per $t=.1 \mathrm{sec})$ reinforcement schedule in $S-$. VI $30 \mathrm{sec}$ remained in effect in $\mathrm{S}+$. There were seven daily durability sessions.

Second Discrimination. OM and YOKE Ss were retrained on the multiple VI 30-sec VI 30-sec reinforcement schedule for 10 daily sessions. A second discrimination training phase followed in which $S$ assignment to $O M$ and YOKE status was reversed from what it had been in the first discrimination. There was no change in TO vs no-TO assignment. The procedure in the 30 daily sessions of the second discrimination was identical to the first.

Second Durability Test. A second durability test, identical to the first, took place next. S assignment to EXT or VT 30-sec status in $S_{-}$- was reversed from what it had been in the first durability test.

\section{RESULTS}

\section{Baseline Training}

Table 1 gives group means and standard deviations of responses per minute in $\mathrm{S}+$ and $\mathrm{S}$ - averaged for each $\mathrm{S}$ over the last 3 days of baseline training. An analysis of variance of these data revealed significantly higher response rates with TO than with no TO $(F=23.64$, $\mathrm{df}=1 / 18, \mathrm{p}<.01)$. A similar rate increase has been reported as a result of increasing TO duration (Mackintosh, Little, \& Lord, 1972; Taus \& Hearst, 
Table 1

Mean Responses Per Minute at the End of Baseline Training

\begin{tabular}{|c|c|c|c|c|c|c|c|c|}
\hline \multirow[b]{3}{*}{ Group } & \multicolumn{4}{|c|}{ No TO } & \multicolumn{4}{|c|}{ TO } \\
\hline & \multicolumn{2}{|c|}{$S^{+}$} & \multicolumn{2}{|c|}{ S- } & \multicolumn{2}{|c|}{ S+ } & \multicolumn{2}{|c|}{ S- } \\
\hline & Mean & SD & Mean & SD & Mean & SD & Mean & SD \\
\hline OM & 30.6 & 8.3 & 29.3 & 7.0 & 59.8 & 4.9 & 56.0 & 9.0 \\
\hline YOKE & 28.1 & 6.1 & 26.0 & 5.1 & 39.2 & 7.8 & 37.6 & 5.4 \\
\hline EXT & 28.9 & 11.4 & 27.2 & 12.1 & 44.8 & 8.4 & 44.3 & 9.2 \\
\hline
\end{tabular}

1970). Response rate was higher in $S+$ than in $D-$ $(\mathrm{F}=4.88, \mathrm{df}=1 / 18, \mathrm{p}<.05)$. This effect probably was due to the houselight being off in S+ and on in S- (this was done to make it possible to observe the rats in $S-$ in subsequent phases of the experiment). There were no other significant sources of variance.

\section{Discrimination Training}

All response rate data reported below were transformed by the shape function method proposed by Anderson (1963) to obtain a measure of relative rate. The transformation treated a rat's responses per minute in $\mathrm{S}+$ or $\mathrm{S}-$ as a proportion of its response rate in the corresponding stimulus component in the last 3 days of baseline training (see Table 1). A unitary relative rate represents responding at the same rate as in baseline training, and a zero relative rate represents cessation of responding. This measure helps to correct for group differences in baseline training as well as reduce within-group variance.

S- Performance. Relative response rate in S- (OM, YOKE, or EXT component) averaged over 3-day blocks was entered into a 3 by 2 by 10 analysis of variance, incorporating OM, YOKE, and EXT conditions, TO vs no TO, and 10 3-day blocks of training, respectively. Group means as a function of blocks of training are shown by the unfilled points in the lower half of Fig. 1 . There was a significant main effect of OM, YOKE, and EXT $(F=7.35, \mathrm{df}=2 / 18, p<.01)$ and a significant linear component of the interaction of $\mathrm{OM}, \mathrm{YOKE}$, and EXT with blocks of training $(\mathrm{F}=10.88, \mathrm{df}=2 / 162$, $\mathrm{p}<.01)$. Decrements in $\mathrm{S}-$ responding occurred more rapidly with OM than with EXT or YOKE, although the linear component of the interaction reflects diminishment in this difference as training progressed. A Newman-Keuls test of the main effect of groups showed that $\mathrm{OM}$ responded significantly less than EXT and YOKE $(p<.05)$ which did not differ from each other.

There was less responding in $\mathrm{S}-$ with $\mathrm{TO}$ than with no TO as reflected in a significant main effect of TO vs no TO $(\mathrm{F}=11.43, \mathrm{df}=1 / 18, \mathrm{p}<.01)$ and a quadratic component of the interaction of TO with blocks of training $(F=11.11, \mathrm{df}=1 / 162, \mathrm{p}<.01)$. Table 2 gives the TO and no-TO group means over blocks of training. The quadratic interaction was due to an increasing difference in response rate until midway through training, when the difference began to diminish to the end of training. The TO variable did not interact with the S- reinforcement schedule. There were no other significant sources of variance except for the main effect of blocks of training, reflecting reduced $\mathrm{S}$ - responding as training progressed.

The mean number of reinforcers received per session in S- by OM Ss (and their yoked controls) over successive 3-day blocks of training were 14.5, 37.0, 67.1, 91.6, 100.4, 96.9, 104.0, 106.1, 108.0, and 111.1. If responding ceased entirely in $S_{-}, 120$ reinforcers per session could be expected on the OM schedule. The number of reinforcers actually received near the end of training compares favorably with this expectation. Also note that these numbers compare with an expected 40 reinforcers per session in $\mathrm{S}+$.

S+ Performance. Analysis of relative response rate in $\mathrm{S}+$ (VI 30-sec component) followed the same design used for S- relative rate. The filled points in the upper half of Fig. 1 show group means in S+ as a function of blocks of training. There was a significant main effect of $S-$ reinforcement schedule $(F=12.72$, df $=2 / 18$, $\mathrm{p}<.01)$. A Newman-Keuls test of the main effect of groups showed that EXT responded significantly more than OM or YOKE $(p<.01)$, which did not differ from

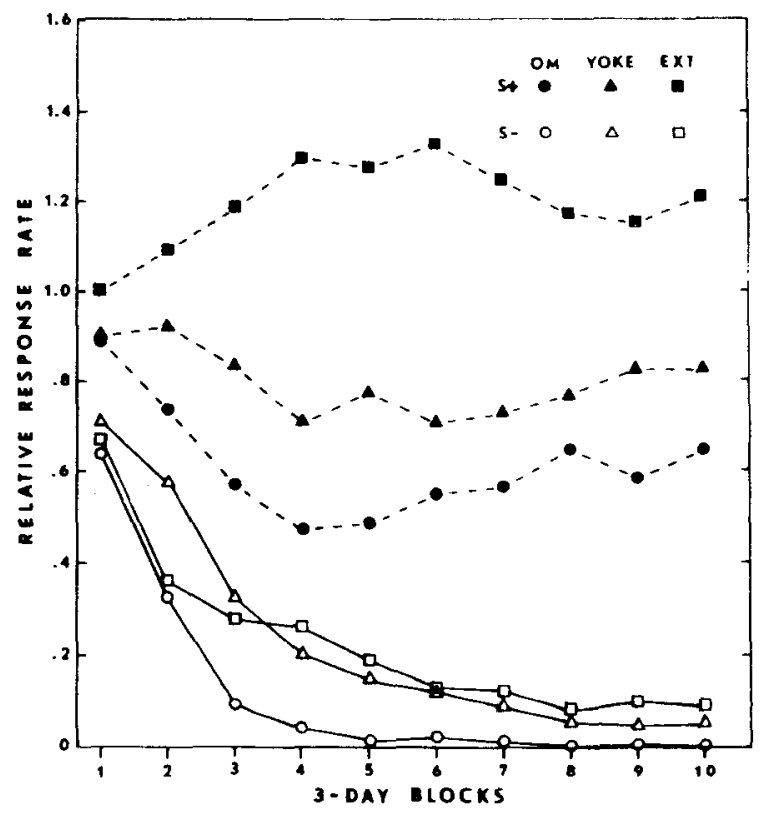

Fig. 1. Group means of relative response rate in $S+$ and $S-$ as a function of 3-day blocks of discrimination training. 
Table 2

TO and No TO Group Means of $S-$ Relative Response Rate During Discrimination Training

\begin{tabular}{lccccccccccc} 
& \multicolumn{10}{c}{ 3-Day Blocks of Training } \\
\cline { 2 - 11 } Group & 1 & 2 & 3 & 4 & 5 & 6 & 7 & 8 & 9 & 10 \\
\hline No TO & .68 & .47 & .32 & .26 & .18 & .15 & .11 & .07 & .08 & .07 \\
TO & .66 & .37 & .14 & .07 & .05 & .04 & .04 & .03 & .02 & .02 \\
\hline
\end{tabular}

each other. However, TO vs no TO interacted with the S- reinforcement schedule $(\mathrm{F}=3.58, \mathrm{df}=2 / 18$, $\mathrm{p}<.05$ ), which is illustrated by the group means in Table 3. Although the TO variable had no sizeable effect in the OM and YOKE groups, response rate in St with EXT was elevated well above baseline without a TO but did not differ from baseline with a TO. Thus, the behavioral contrast effect often found in multiple VI EXT schedules was only obtained in the present experiment when there was no TO between stimulus changes. There were no other significant sources of variance.

\section{Durability Testing}

Figure 2 shows OM, YOKE, and EXT group means of relative response rate as a function of daily sessions; the left panel shows results in the VT durability test and the right panel shows results in EXT. These data were entered into four separate 2 by 3 by 7 analyses of variance, one for each combination of stimulus component and durability test procedure, incorporating TO vs no TO, OM, YOKE, or EXT discrimination training, and seven daily sessions, respectively. Evaluation of the durability data requires examination of changes from terminal discrimination performance (see Fig. 1) as well as comparisons between groups during durability testing.

S- Rate with VT in S-. Group means of relative Srate are shown in the lower three curves in the left panel of Fig. 2. Groups EXT and YOKE increased responding at higher rates than $O M$ on the first 4 days, after which EXT and YOKE responding declined to the low level maintained by OM throughout the durability test. This effect was represented in a significant linear componenet of the interaction of OM, YOKE, and EXT with sessions $(\mathrm{F}=7.15, \mathrm{df}=2 / 36, \mathrm{p}<.01)$. There were no other significant sources in the analysis of these data. Despite the statistically significant interaction, it should be noted that all groups exhibited considerable durability of response elimination, especially in comparison to the EXT durability test results shown in the right panel of Fig. 2.

\section{Table 3}

S+ Mean Relative Rates for the Interaction of $S-$ Reinforcement Schedule by TO vs No TO

\begin{tabular}{lccr}
\hline & OM & YOKE & EXT \\
\hline No TO & .55 & .79 & 1.43 \\
TO & .67 & .81 & .96 \\
\hline
\end{tabular}

S+ Rate with VT in S-. The upper three curves in the left panel of Fig. 2 show groups means in S+. Group OM responded at lower rates than did YOKE or EXT throughout the durability test. This effect was represented in a significant main effect of groups $(F=5.75$, df $=2 / 6, p<.05)$, a Newman-Keuls test showed that $\mathrm{OM}$ responded at significantly lower rates than did YOKE or EXT $(p<.05)$, which did not differ from each other. There also was a significant linear component of the interaction of OM, YOKE, and EXT with sessions $(\mathrm{F}=10.05, \mathrm{df}=2 / 36, \mathrm{p}<.01)$, which can be attributed to the steady decline in response rate in EXT over sessions. OM response rate remained at the same low level exhibited at the end of training. YOKE response rate shifted abruptly upward from its level at the end of training and remained elevated throughout the durability test.

S- Rate with EXT in $\mathbf{S}-$. Group means of relative response rate in $\mathbf{S}$ - are shown in the lower three curves in the right panel of Fig. 2. Groups OM and YOKE both resumed responding at substantial rates when reinforcement was no longer forthcoming in $\mathrm{S}_{-}^{-}$, while Group EXT continued to respond at low rates. This effect was demonstrated in a significant main effect of OM, YOKE, and EXT ( F = 14.09, df $=2 / 6, p<.01)$, and a Newman-Keuls test showed that EXT responded less than OM, which responded less than YOKE $(p<.05)$.

S+ Rate with EXT in S-. The upper three curves in the right panel of Fig. 2 shows group means of relative response rate in $\mathrm{S}+$. There were no significant sources in the analysis of variance. However, compared with terminal discrimination performance, there were dramatic increases in $\mathrm{S}+$ rate in Groups $\mathrm{OM}$ and YOKE from a level well below baseline to $150 \%$ of baseline when EXT was introduced in S-. This demonstrates an immediate behavioral contrast effect in Groups OM and YOKE.

\section{OM vs YOKE Within Subjects}

S-- Discrimination Performance. Relative response rate in $\mathrm{S}-$ from the two series of discrimination training was entered into a 2 by 2 by 2 by 10 analysis of variance incorporating, resprectively: (a) TO vs no TO, (b) order of OM and YOKE status, (c) OM vs YOKE, and (d) 10 3-day blocks of training. Relative response rate in the second discrimination was based on response rate averaged over the last 3 days of retraining. The lower four curves in Fig. 3 show the group means for OM vs YOKE and order of OM and YOKE status as a function 


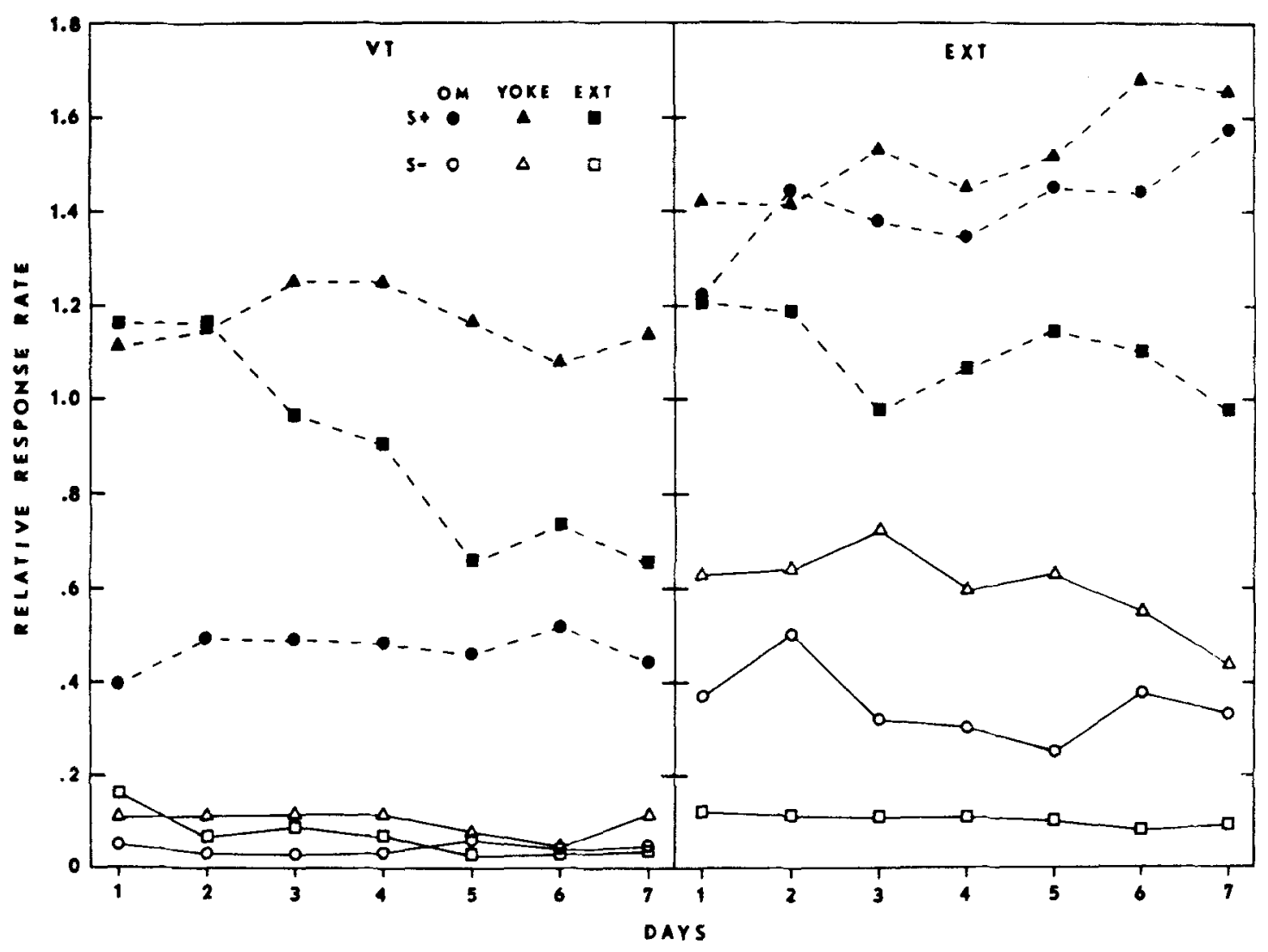

Fig. 2. Group means of relative response rate in $S+$ and $S-$ as a function of days of durability testing. The left panel represents the VT durability test, and the right panel represents the EXT durability test.

of blocks of training. The result of principal interest is subsumed in the significant linear component of the interaction of OM vs YOKE with order of OM and YOKE status with blocks of training $(F=38.50$, $\mathrm{df}=1 / 108, \mathrm{p}<.01$ ). Although response elimination was more rapid with OM than with YOKE in both discrimination training series, this effect was much larger in the first discrimination than in the second. The involvement of blocks of training in this triple interaction simply reflects diminishment in these differences as training progressed. Of course, there were significant main effects of $O M$ vs YOKE $(F=22.11$, $\mathrm{df}=1 / 12, \mathrm{p}<.01)$ and order of $\mathrm{OM}$ and YOKE status $(F=6.67, \mathrm{df}=1 / 12, \mathrm{p}<.05)$. The latter effect was due to less responding with the OM YOKE order than with YOKE-OM. There were no other significant sources in this analysis.
The median delay of reinforcement following a response was computed in every session of both discrimination training series for each OM and YOKE S. The length of the delay represents the extent to which responding might have been adventitiously reinforced. These data were entered into an analysis of variance of the same design used for relative response rate above, except that only the first five blocks of training were included (OM Ss did not respond enough in the last half of training to obtain reliable medians). Table 4 gives means for Group OM in the first five blocks of training and for Group YOKE in all 10 blocks. Delay of reinforcement was longer with OM than with YOKE at the outset of training, and OM delays increased rapidly in succeeding blocks while YOKE delays gradually lengthened. This result was reflected in a significant linear component of the interaction of OM vs YOKE

Table 4

Group Means of Median Delay of Reinforcement

\begin{tabular}{llrrrrrrrrrr}
\hline & & \multicolumn{10}{c}{ Blocks of 3 Days } \\
\cline { 2 - 12 } Order & Group & 1 & 2 & 3 & 4 & 5 & 6 & 7 & 8 & 9 & 10 \\
\hline \multirow{2}{*}{ OM-YOKE } & OM & 4.7 & 7.0 & 10.0 & 15.2 & 19.3 & & & & & \\
& YOKE & 2.7 & 5.6 & 9.1 & 9.7 & 10.9 & 10.5 & 7.3 & 5.0 & 7.9 & 7.8 \\
\multirow{2}{*}{ YOKE-OM } & OM & 6.5 & 10.3 & 19.4 & 24.4 & 20.2 & & & & & \\
& YOKE & 1.9 & 2.4 & 3.8 & 4.1 & 3.6 & 5.0 & 7.0 & 5.0 & 7.7 & 7.7 \\
\hline
\end{tabular}


with blocks of training $(\mathrm{F}=11.48, \mathrm{df}=1 / 48, \mathrm{p}<.01)$ and a significant main effect of $\mathrm{OM}$ vs YOKE $(\mathrm{F}=64.45, \mathrm{df}=1 / 12, \mathrm{p}<.01)$. Also, there was an interaction of $\mathrm{OM}$ vs YOKE with order of $\mathrm{OM}$ and YOKE status $(\mathrm{F}=20.47, \mathrm{df}=1 / 12, \mathrm{p}<.01)$ which was due to longer delays in the second discrimination, especially in the case of the YOKE condition. It should be noted in Table 4 that Group OM's mean median delays were less than the $30 \mathrm{sec}$ expected with the variable postresponse interval. This was due to the random selection of a new postresponse interval after every response. In the first half of training, $\mathrm{OM}$ rats responded at sufficient rates to frequently interrupt longer postresponse intervals before they timed out. Therefore, postresponse reinforcement tended to occur with shorter intervals which timed out before interruption by a response.

S+ Discrimination Performance. The upper two curves in Fig. 3 show OM and YOKE group means of $\mathrm{S}+$ relative response rate as a function of blocks of training. Although both groups responded below baseline levels, $\mathrm{OM}$ responded at lower rates than YOKE $(\mathrm{F}=7.28$, $\mathrm{df}=1 / 12, \mathrm{p}<.05)$. There were no other significant sources of variance in the analysis of the same design used for $\mathrm{S}$ - relative rate.

Durability Testing. Within-Ss comparisons of OM vs

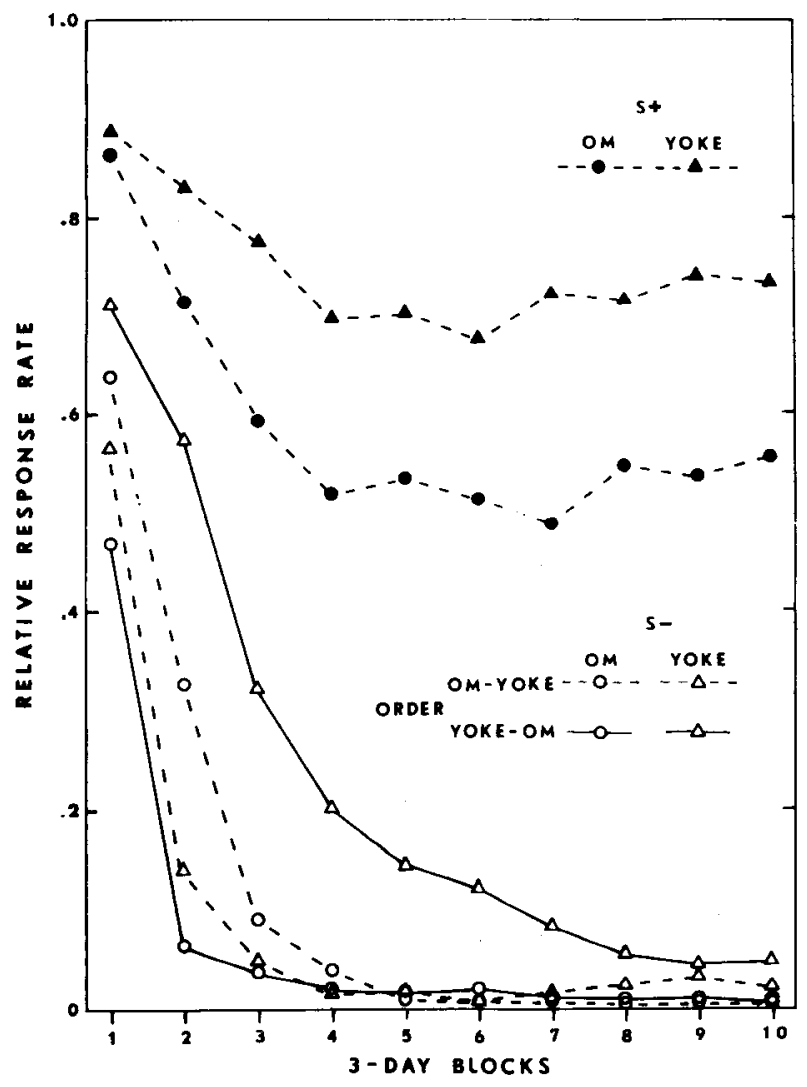

Fig. 3. Group means of relative response rate in $S+$ and $S-$ as a function of 3-day blocks of training collapsed over two discrimination training series. S- includes separate group means for the two orders of OM and YOKE status.
YOKE relative response rate were made in four separate analyses of variance, one for each combination of stimulus component and durability test procedure, of the same general design used for discrimination performance. These results were consistent with those reported for the first durability test and need not be reported in any further detail.

\section{DISCUSSION}

Two comparisons which require separate discussion are $\mathrm{OM}$ vs EXT and $\mathrm{OM}$ vs YOKE. Response elimination in $\mathrm{S}-$ of the multiple VIEXT or VIOM was considerably more rapid with OM than EXT. This finding is in agreement with similar comparisons made by Nevin (1968), Sherman (1971), and Snow and Uhl (1968). However, comparisons of OM and EXT in nondiscriminative procedures generally have failed to demonstrate more effective response elimination with $\mathrm{OM}$, and several have found EXT to be more effective than OM (Harman, 1973; Uhl, 1973; Uhl \& Garcia, 1969; Uhl \& Sherman, 1971). It is possible that the present result is a characteristic of multiple schedules in which a VI reinforcement schedule in $\mathrm{S}+$ is to be discriminated from OM or EXT in $\mathrm{S}-$. In another related study, Zeiler (1971) found OM to be a more effective response eliminator than EXT in a multiple schedule, within-Ss comparison. Another factor which may have enhanced $O M$ effectiveness was the use of variable $O M$ intervals in the present experiment (also Nevin, 1968). All of the available OM vs EXT nondiscriminative comparisons have used fixed OM intervals. Uhl (1974) has shown that variable OM intervals are more effective than fixed intervals. However, the possibility remains that fixed $O M$ intervals might be equally, or more, effective than variable intervals when a VI operates in St since the variable vs fixed temporal distribution of reinforcers would constitute an additional discriminative dimension (cf. Jenkins, 1965).

Increases in response rate in $\mathrm{S}+$ above baseline rates were obtained when EXT was in effect in S- and there was no TO between stimulus changes. This is a demonstration of the well-known behavioral contrast effect (cf. Bloomfield, 1969). The effect of the TO variable was similar to those reported by Mackintosh, Little, and Lord (1972). They reported that contrast only occurred when the TO was short, but did not occur with a long TO. On the other hand, S+ response rates with $\mathrm{OM}$ in $\mathrm{S}$ - declined to $50 \% .60 \%$ of baseline rates. These results, together with Nevin (1968), stand in clear contradiction to Terrace's (1968) position that a reduction in response rate in $S-$ is sufficient to cause behavioral contrast.

OM proved to be more effective than the YOKE procedure in nearly every possible comparison. Response elimination was more rapid in $\mathrm{S}-$ with $\mathrm{OM}$ than with YOKE in two separate discrimination training series. This finding confirms and extends Davis and Bitterman's (1971) comparison of OM and YOKE in a 
nondiscriminative procedure. However, extensive response elimination was achieved with the YOKE procedure in the present experiment. In fact, EXT and YOKE were equivalent in effectiveness. This raises the question of what process underlies response elimination with the YOKE procedure. Davis and Bitterman suggested that response elimination was retarded with YOKE in comparison to $\mathrm{OM}$ because occasional relatively immediate reinforcement followed responding in the YOKE case. The present data showed that delay of reinforcement was shorter for YOKE than for OM Ss. However, it is unclear why there should have been such extensive reduction in response rate in the YOKE group if delay of reinforcement was the controlling variable. Furthermore, the delays experienced by OM Ss at the outset of discrimination training were short enough to sustain responding if reinforcement had been dependent on responding.

An alternative view advocated by Baum (1973) is that behavior is controlled by the correlation between response rate and reinforcement frequency instead of strict temporal contiguity between an isolated response and the reinforcing event. In a correlational analysis of the present experiment, OM Ss experienced a negative correlation between response rate and reinforcement frequency, thus leading to the elimination of responding in $\mathrm{S}-$. YOKE $\mathrm{Ss}$ experienced a slightly negative correlation early in discrimination training since their response rate had declined through extinction by the time their paired OM Ss began receiving reinforcers (cf. Uhl, 1974), leading to reduction in their responding. By the end of discrimination training, when OM Ss had virtually ceased responding in $\mathrm{S}_{-}$, the correlation for YOKE Ss was zero.

Comparisons of $\mathrm{OM}$ and YOKE performance in $\mathrm{S}+$ during discrimination training revealed considerably more induction of responding with $\mathrm{OM}$ than with YOKE. This result suggests that the learning to inhibit responding in $S-$ due to the response-reinforcement correlation generalized to performance in $\mathrm{S}+$, and that the degree of inhibition in $S$ - was reflected in amount of $\mathrm{S}+$ induction. It is difficult to account for the induction effect on the basis of relative densities of reinforcement in $\mathrm{S}+$ and $\mathrm{S}-$ since the effect appeared immediately at the outset of discrimination training at a time when fewer reinforcers occurred in S- than in St. Also, reinforcement density fails to account for the differences between $\mathrm{OM}$ and YOKE which received exactly the same reinforcement in S-.

The durability test results further emphasize differences in what Groups OM and YOKE had learned during discrimination training. In the VT durability test, involving a two-thirds reduction in reinforcement frequency in the former $S-$, YOKE resumed responding in $\mathrm{S}-$ more than OM. In S+, YOKE displayed a sharp increase in response rate, amounting to behavioral contrast, while OM maintained the induction effect they had shown throughout discrimination training. This contrast effect occurred in Group YOKE despite the equality of reinforcement frequency in the two stimulus components in the VT durability test. In the EXT durability test, both $\mathrm{OM}$ and YOKE exhibited substantial response resumption in $\mathrm{S}-$, although, here again, YOKE effects were less durable than were OM effects. Both OM and YOKE sharply increased response rates amounting to a behavioral contrast effect. The results obtained in $\mathrm{S}+$ in the durability test indicate that St performance may be used as a sensitive index of the effects of the change in the $S-$ reinforcement schedule. The St performance of the OM group gives further evidence that omission training is an effective and durable response elimination procedure.

\section{REFERENCES}

Anderson, N. H. Comparison of different populations: Resistance to extinction and transfer. Psychological Review, $1963,70,162-179$

Baum, W. M. The correlation-based law of effect. Journal of the Experimental A nalysis of Behavior, 1973, 20, 137-153.

Bloomfield, T. M. Behavioral contrast and the peak shift. In $R$. M. Gilbert and N. S. Sutherland (Eds.), Animal discrimination learning. New York: Academic Press, 1969. Pp. 215-241.

Davis, J., \& Bitterman, M. E. Differential reinforcem ent of other behavior (DRO): A yoked-control comparison. Journal of the Experimental A nalysis of Behavior, 1971, 15, 237-241.

Grant, D. A. Classical and operant conditioning. In A. W. Melton (Ed.), Categories of human learning. New York: Academic Press, 1964.

Harman, R. E. Response elimination in concurrent and single operant situations with pigeons. Learning \& Motivation, 1973, 4, 417-431.

Jenkins, H. M. Measurement of stimulus control during discriminative operant conditioning. Psychological Bulletin, $1965,64,365-376$.

Kamil. A. C., \& Davenport, J. W. The role of adventitious reinforcement in operant discrimination. Journal of Experimental Psychology, 1968, 76, 609-617.

Mackintosh, N. J., Little, L., \& Lord, J. Some determinants of behavioral contrast in pigeons and rats. Learning \& Motivation, 1972, 3, 148-161.

Nevin, J. A. Differential reinforcement and stimulus control of not responding. Journal of the Experimental Analysis of Behavior, 1968, 11, 715-726.

Reynolds, G.S. Behavioral contrast. Journal of the Experimental A nalysis of Behavior, $1961,4,57-71$.

Sherman, W. O. Effects on response elimination of omission and extinction with an added counter. Unpublished doctoral extinction with an added counter.
dissertation, University of Utah, 1970.

Smith, J. B., \& Clark, F. C. Two temporal parameters of food postponement. Journal of the Experimental Analsyis of Behavior, 1972, 18, 1-12.

Snow, M. E., \& Uhl, C. N. Effects of omission, extinction, and change-over-delay procedures on free operant discrimination performance. Communications in Behavioral Biology, 1968,5, No. 5.

Terrace, H. S. Discrimination learning, the peak shift, and behavioral contrast. Journal of the Experimental Analysis of Behavior, 1968, 11, 727-741.

Taus, S. E., \& Hearst, E. Effects of intertrial (blackout) duration on response rate to a positive stimulus. Psychonomic Science, $1970,19,265-266$.

Topping, J. S., \& Larmi, O. K. Response elimination effectiveness of omission and two extinction training procedures. Psychological Record, 1973, 23, 197-202.

Topping, J. S., Pickering, J. W., \& Jackson, J. A. Omission training effects following VI and FI pretraining. Psychonomic Science, 1971, 24, 113-114.

Uhl, C. N. Response elimination in rats with schedules of omission training, including yoked and response-ind ependent reinforcement comparisons. Learning \& Motivation, 1974, in press. 
Uhl, C. N. Eliminating behavior with omission and extinction after varying amounts of training. Animal Learning \& Behavior, 1973, 1, 237-240.

Uhl, C. N., \& Garcia, E, E. Comparison of omission with extinction in response elimination in rats. Journal of Comparative \& Physiological Psychology, 1969, 69, 554-562.

Uhl, C. N., \& Sherman, W. O Comparison of combinations of omission, punishment, and extinction methods in response elimination in rats. Journal of Comparative \& Physiological Psychology, 1971, 74, 59-65.
Weisman, R, G, Factors influencing inhibitory stimulus control: $D$ ifferential reinforcement of other behavior during discrimination training. Journal of the Experimental Analysis of Behavior, 1970, 14, 87-91.

Zeiler, M. D. Eliminating behavior with reinforcement. Journal of the Experimental Analysis of Behavior, 1971, 16, 401-405.

(R eceived for publication April 8, 1974; accepted July 11, 1974.) 